\title{
Efficacy and safety of consolidation chemotherapy during the resting period in patients with local advanced rectal cancer
}

\author{
HUAN-QING LIANG ${ }^{1,2 *}$, ZHONG-YI DONG ${ }^{1 *}$, ZHI-JIE LIU ${ }^{3}$, JIAO LUO $^{4}$, \\ QIN ZENG $^{1}$, PING-YAN LIAO ${ }^{1}$ and DE-HUA WU ${ }^{1}$
}

\begin{abstract}
${ }^{1}$ Department of Radiation Oncology, Nanfang Hospital, Southern Medical University, Guangzhou, Guangdong 510515; ${ }^{2}$ Department of Radiation Oncology, Dongguan People's Hospital, Dongguan, Guangdong 523000; ${ }^{3}$ Department of Radiation Oncology, Cancer Institute of Guangxi Zhuang Autonomous Region, The Affiliated Cancer Hospital of Guangxi Medical University, Nanning, Guangxi 530021; ${ }^{4}$ Department of Pathology, Nanfang Hospital, Southern Medical University, Guangzhou, Guangdong 510515, P.R. China
\end{abstract}

Received February 4, 2018; Accepted September 11, 2018

DOI: $10.3892 / \mathrm{ol} .2018 .9804$

\begin{abstract}
It remains controversial as to whether a long interval between neoadjuvant chemoradiotherapy (NCRT) and surgery may provide clinical benefits for patients with local advanced rectal cancer (LARC). The addition of consolidation chemotherapy during the resting period was recently considered as a treatment option. The present study aimed to verify the efficacy and safety of consolidation chemotherapy during the resting period in patients with LARC. A total of 156 patients with local advanced stage T3-4N0-2 rectal cancer were enrolled between January 2010 and July 2016. Patients were divided into two groups, those who received consolidation chemotherapy prior to surgery $(n=76)$ and the control group who did not $(n=80)$. Multivariate logistic regression and the Kaplan-Meier method were used to explore the predictors of pathological complete response (pCR) and survival. The demographic and tumor characteristics were comparable
\end{abstract}

Correspondence to: Dr De-Hua Wu, Department of Radiation Oncology, Nanfang Hospital, Southern Medical University, 1838 North of Guangzhou Avenue, Guangzhou, Guangdong 510515, P.R. China

E-mail: 18602062748@163.com

*Contributed equally

Abbreviations: NCRT, neoadjuvant chemoradiotherapy; LARC, local advanced rectal cancer; NCCN, National Comprehensive Cancer Network; pCR, pathological complete response; OS, overall survival; DFS, disease-free survival; DMFS, distant metastases-free survival; TME, total mesorectal excision; BMI, body mass index; AJCC, American Joint Committee on Cancer; CEA, carcinoembryonic antigen; TN, tumor-node; UICC, Union for International Cancer Control

Key words: consolidation chemotherapy, pCR, near pCR rate, DFS, LARC between the two groups. The consolidation group yielded significantly higher $\mathrm{pCR}$ and near $\mathrm{pCR}$ rates compared with the control group $(\mathrm{P}=0.015)$. Patients in the consolidation group who also underwent standard adjuvant chemotherapy displayed improved 3-year disease-free survival (DFS) compared with the control group $(\mathrm{P}=0.036)$. Notably, the addition of consolidation chemotherapy between NCRT and surgery did not significantly increase the incidence of surgical complications and grade 3 or 4 toxicities when compared with the control group. Consolidation chemotherapy was associated with increased $\mathrm{pCR} /$ near pCR rates and improved 3-year DFS, and displayed a manageable safety profile. The present study provided primary evidence for the efficacy and safety of consolidation chemotherapy in LARC. Further prospective studies are warranted in the future to verify these results.

\section{Introduction}

Globally, the incidence of colorectal cancer ranks as the fifth highest of all malignant tumor types. Cancer mortality caused by colorectal cancer accounts for the sixth highest cancer mortality rate globally (1). One previous study reported that the incidence of rectal cancer accounts for $\sim 40 \%$ of the total incidence of colorectal cancer, and that there is a rising trend (2). Locally advanced rectal cancer constitutes up to $50 \%$ of all rectal cancer types and includes nonresectable and borderline-resectable tumor types (3). Currently, neoadjuvant chemoradiotherapy (NCRT) combined with postoperative chemotherapy, or neoadjuvant chemotherapy combined with postoperative chemoradiotherapy, are the standard treatments for local advanced rectal cancer (LARC) in the National Comprehensive Cancer Network (NCCN) guidelines (4).

Pathological complete response (pCR) following NCRT is an important prognostic indicator for patients with excellent treatment outcomes $(5,6)$. Tumor regression occurs slowly. Prolonging treatment intervals in rectal cancer may increase the $\mathrm{pCR}$ of the patient, $\mathrm{R} 0$ resection, and anus preservation rates $(7,8)$. To achieve an improved $\mathrm{pCR}$ rate, a number of studies recommend an interval of 7-8 weeks between NCRT 
and surgery (7-9). However, the high pCR rate obtained from long intervals between NCRT and surgery is not associated with a survival benefit, with long and short treatment intervals conferring a similar rate of overall survival (OS) (10-12). Notably, Supiot et al (13) reported that OS and recurrence-free survival (RFS) were slightly lower in the long-interval group compared with those in the short-interval group, which may be due to the prolonged resting period that may increase the probability of distant metastases. This occurrence is more common in ypT3-4N0 and ypT0-4N+ patients who have high risk of distant metastases.

Preoperative chemotherapy may improve patient tolerance to chemotherapy and reduce the incidence of distant metastases. Therefore, theoretically, consolidation chemotherapy between NCRT and surgery may increase the rate of OS and DFS, in addition to distant metastases-free survival (DMFS). Although no corresponding guidelines have been established to date, consolidation chemotherapy is gradually attracting the attention of the research community.

In the present study, the impact of adding consolidation chemotherapy for rectal cancer during the resting period was retrospectively analyzed in order to determine whether consolidation chemotherapy resulted in higher rates of $\mathrm{pCR}$ and near $\mathrm{pCR}$ and improved survival, and to assess the influence of consolidation chemotherapy on chemotherapy toxicities, surgical outcomes and postoperative complications.

\section{Patients and methods}

Patients. A total of 273 patients with cT3-4 N0-2 M0 LARC who underwent neoadjuvant chemoradiation followed by radical resection with total mesorectal excision (TME) at Nanfang Hospital of Southern Medical University (Guangzhou, China) were reviewed. The selection criteria were: i) Age $\leq 80$ years; ii) cT3-4 N0-2 M0 LARC; iii) tumor located 0-10 cm from anal verge; iv) underwent neoadjuvant chemoradiation followed by TME; v) chemotherapeutic regimen was capecitabine, CapeOX or FOLFOX; and vi) dose of radiotherapy was 45-50.4 Gy. Of these patients, 117 were excluded due to age $>80$ years $(\mathrm{n}=7)$, tumor located $>10 \mathrm{~cm}$ from the anal verge $(\mathrm{n}=23)$, or ineligibility due to either chemotherapeutic regimen $(n=54)$ or radiotherapy (not 45-50.4 Gy; n=33). Overall, 156 patients were included in the analysis. Patients were divided into the following two groups according to whether they received chemotherapy or not: Consolidation $(n=76)$ or control $(n=80)$ group (Fig. 1). The following data were reviewed: Sex, age, body mass index (BMI), pretreatment level of carcinoembryonic antigen (CEA), histological type, clinical Tumor, Nodule, Metastasis (TNM) stages graded according to the American Joint Committee on Cancer (AJCC), 7th edition guidelines (14), pretreatment distance of the tumor from the anal verge, NCRT regimen, interval between NCRT and surgery, grades 3 or 4 toxicities, final pathological stage, postoperative complications, disease recurrence, distant metastases, and mortality. The tumor histology and TNM stages of patients were confirmed by at least two relevant experts. Pathological reports were reviewed to assess tumor-node $(\mathrm{TN})$ downstaging and the pCR and near pCR rates. Near pCR was defined as a finding of only isolated residual tumor cells or small groups of residual cancer cells in the rectal wall, with no tumor cells in the lymph nodes $(9,15)$. Chemotherapy regimens included capecitabine $\left(825 \mathrm{mg} / \mathrm{m}^{2}\right.$ twice daily, 5 days/week for 5 weeks for concurrent chemoradiotherapy; $1,250 \mathrm{mg} / \mathrm{m}^{2}$ twice daily on days 1-14, every 3 weeks for consolidation chemotherapy and postoperative therapy), CapeOX (oxaliplatin $130 \mathrm{mg} / \mathrm{m}^{2} \mathrm{IV}$, day 1 ; capecitabine $1,000 \mathrm{mg} / \mathrm{m}^{2}$ twice daily on days 1-14/3 weeks), or FOLFOX (oxaliplatin $85 \mathrm{mg} / \mathrm{m}^{2}$ IV, day 1 ; leucovorin $400 \mathrm{mg} / \mathrm{m}^{2}$ IV on days $1-2$, and $5-\mathrm{FU}$ $400 \mathrm{mg} / \mathrm{m}^{2}$ IV bolus on day 1 followed by continuous infusion of $2,400 \mathrm{mg} / \mathrm{m}^{2}$ over $46-48 \mathrm{~h}$, repeated every 2 weeks). The actual cycles of consolidation chemotherapy varied due to numerous reasons, including tumor response, clinician decision, and patient economic status. The mean radiation dose was $50.4 \mathrm{~Gy}$ (range, 45-50.4 Gy) with a daily fraction of $1.8 \mathrm{~Gy}$ using a $6-\mathrm{mV}$ linear accelerator. Surgery was performed approximately 6-8 weeks following the completion of preoperative therapy, irrespective of clinical tumor stage or response to NCRT. All the patients underwent curative resection with TME. The Ethics Committee of Nanfang Hospital of Southern Medical University approved the present study.

Statistical analysis. Continuous variables are expressed as the median and range and were analyzed using two-sample t-tests. Categorical variables are expressed as numbers with percentages and were analyzed using a $\chi^{2}$ or Fisher's exact test when appropriate. Univariate analysis was used to preliminarily assess the predictive value of variables for achieving pCR and near pCR. Multivariate analysis was performed on variables with $\mathrm{P}<0.1$ from the univariate analysis to further analyze the independent predictive factors of $\mathrm{pCR}$ and near $\mathrm{pCR}$. All analyses were performed using SPSS 21.0 software (IBM Corp., Armonk NY, USA) for Windows. OS was calculated from the date of surgery to the date of mortality from any cause or the last follow-up visit. Recurrence was determined either via imaging studies or pathological findings. DMFS was defined as the time from surgery to metastasis or mortality, while DFS was defined as the time from surgery to local recurrence, metastasis or mortality. The Kaplan-Meier method was used to analyze the rate of patient survival, and comparisons were performed using the Wilcoxon test. All statistical tests were two-sided. $\mathrm{P}<0.05$ was considered to indicate a statistically significant difference.

\section{Results}

Clinicopathological characteristics. Between January 2010 and July 2016, 156 patients with local advanced (cT3-4 and/or $\mathrm{cN} 0-2)$, low $(0-5 \mathrm{~cm}$ from the anal verge), and mid- $(6-10 \mathrm{~cm}$ from the anal verge) rectal cancer were enrolled in the present study. The baseline characteristics of the patients were well balanced between the two groups (Table I). Abdominopelvic enhanced magnetic resonance imaging (MRI) was performed on all patients prior to treatment, and 148 (94.8\%) patients underwent endoscopic ultrasonography. The median age at diagnosis was 53 years (range, 22-80 years). The majority of the patients were male (69.2\%), while females comprised $30.8 \%$ of the study population. The majority of tumors were staged as cT4 (76.3\%) and III (83.3\%). A total of $90(57.7 \%)$ and $60(42.3 \%)$ patients had a tumor located either between 0 and $5 \mathrm{~cm}$, or 6 and $10 \mathrm{~cm}$, respectively, from the anal verge. No differences 
273 Evaluable patients with T3-4NO-2 rectal cance assessed by MRI and/or endorectal ultrasound

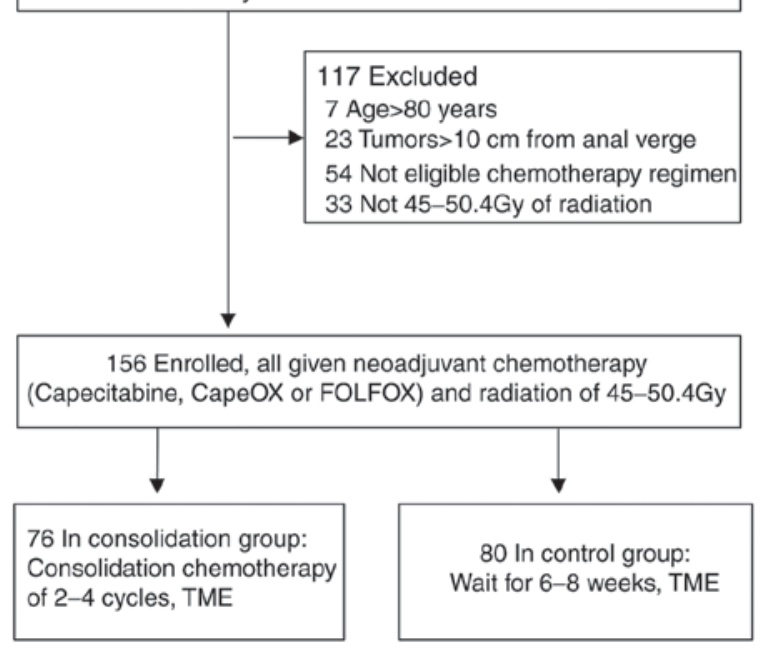

Figure 1. Study flow chart. A total of 273 patients were initially evaluated to take part in the study. A total of 117 of these were excluded, resulting in a final total of 156 patients enrolled on to the study. These were split into the consolidation group $(n=76)$ and the control group $(n=80)$. MRI, magnetic resonance imaging; TME, total mesorectal excision.

in stage were observed between the two groups $(\mathrm{P}=0.567)$. The median interval between NCRT and TME was 8.7 weeks (range, 5-12 weeks) in the consolidation group and 7.4 weeks (range, 2.4-12.2 weeks) in the control group ( $\mathrm{P}=0.17)$. A total of $89(57.1 \%)$ patients had an interval of $\geq 8$ weeks, while 67 $(42.9 \%)$ had an interval of $<8$ weeks. The majority of patients $(64,84.2 \%)$ received consolidation chemotherapy throughout the entire resting period. The median interval between NCRT and TME was 8.7 weeks in the consolidation group. The majority of patients received two cycles of capecitabine (40/42) or CapeOX (9/14), while 7/20 patients received three cycles of FOLFOX during the resting period. Due to a prolonged resting period caused by swelling of the rectum and surrounding tissues, 2/42 and 1/14 patients were administered three cycles of capecitabine and CapeOX, respectively, and 5/20 patients administered four cycles of FOLFOX. All patients underwent pelvic enhancement MRI following neoadjuvant chemoradiation. MRI scans were reviewed by a radiologist with $>5$ years of experience in the MRI assessment of rectal cancer, using MERCURY Experience defined criteria (16). Levels of mrTRG-4 and mrTRG-5 correspond to a response to concurrent chemotherapy, hence the clinician decided to administer only one cycle of consolidation chemotherapy to these patients (2/14 in CapeOX and 2/20 in FOLFOX). Due to economic constraints, $2 / 14$ patients were given one cycle of CapeOX and 6/20 patients given two cycles of FOLFOX. All patients underwent optimal surgery with TME, among which $36(47.4 \%)$ patients in the consolidation group and $33(41.2 \%)$ patients in the control group received $>3$ months of adjuvant chemotherapy following surgery. The median follow-up time was 31 months (range, 4-84 months).

pCR and near PCR. A total of 38/156 (24.3\%) patients had a pCR or near pCR. Among them, a total of 16 (21.1\%) patients in the consolidation group and nine (11.25\%) patients in the control group achieved pCR, while nine (11.84\%) patients in the consolidation group and four (5\%) patients in the control group achieved near pCR. The rate of $\mathrm{pCR}$ and near $\mathrm{pCR}$ was significantly higher in patients who underwent consolidation chemotherapy $(25 / 76,32.8 \%)$ compared with that in patients who underwent NCRT only $(13 / 80,16.25 \%),(\mathrm{P}=0.015)$. In the univariate analysis, age, sex, differentiation, distance from the anorectal verge, lymphovascular invasion and BMI were not associated with $\mathrm{pCR}$ and near $\mathrm{pCR}$ rates $(\mathrm{P}=0.732,0.780$, $0.424,0.139,0.250$ and 0.919 , respectively). Clinical $\mathrm{T}$ stage (T3 vs. $\mathrm{T} 4, \mathrm{P}=0.002$ ), $\mathrm{N}$ stage (N0 vs. $\mathrm{N} 1$ vs. $\mathrm{N} 2, \mathrm{P}=0.012$ ), AJCC/Union for International Cancer Control (UICC) stage (II vs. III, $\mathrm{P}=0.005), \mathrm{CEA}$ level $(<5 \mathrm{ng} / \mathrm{ml}$ vs. $\geq 5 \mathrm{ng} /$ $\mathrm{ml}, \mathrm{P}=0.014$ ), neural invasion (yes vs. no, $\mathrm{P}=0.064$ ), interval between NCRT and TME ( $\geq 8$ weeks vs. $<8$ weeks, $\mathrm{P}=0.000$ ), and given consolidation chemotherapy (yes vs. no, $\mathrm{P}=0.015$ ) were significantly associated with $\mathrm{pCR}$ and near $\mathrm{pCR}$ rates. Multivariate analysis was performed for the characteristics that had $\mathrm{P}<0.1$ in the univariate analysis (Table II). Aside from neural invasion [OR: $0.447 ; 95 \%$ confidence interval (CI): 0.070-2.838; $\mathrm{P}=0.393$ ], $\mathrm{N}$ stage (OR: 0.729; 95\% CI: $0.280-1.902 ; \mathrm{P}=0.518$ ), and AJCC/UICC stage (OR: 0.260; 95\% CI: 0.041-1.661; $\mathrm{P}=0.155)$, clinical T stage (OR: 0.273; 95\% CI: 0.110-0.677; P=0.005), CEA level (OR: 0.378, 95\% CI: 0.151-0.944; $\mathrm{P}=0.037$ ), interval between CRT and TME (OR: 0.263 ; 95\% CI: $0.088-0.785 ; \mathrm{P}=0.017)$, and given consolidation chemotherapy (OR: 0.378; 95\% CI: 0.151-0.944; $\mathrm{P}=0.037$ ) were factors significantly associated with $\mathrm{pCR}$ and near $\mathrm{pCR}$.

Survival. The Kaplan-Meier curves (Fig. 2) demonstrated that the 3-year DMFS (70.72\% vs. 74.64\%; HR: 1.129; 95\% CI: $0.5928-2.162 ; \mathrm{P}=0.70)$, DFS (70.95\% vs. $71.89 \%$; HR: 1.063 ; 95\% CI: $0.5801-1.952 ; \mathrm{P}=0.84)$, and OS $(73.99 \%$ vs. $83.40 \%$; HR: 1.286 ; 95\% CI: 0.6300-2.666; $\mathrm{P}=0.48$ ) did not differ significantly between the two groups. The decision for adjuvant treatment was made on an individual basis according to pathological staging. A total of $69(44.23 \%)$ patients received adjuvant chemotherapy for $>3$ months, implementing the same regimen used for neoadjuvant chemotherapy (36 in the consolidation group and 33 in the control group). The survival rate of the 69 patients was further analyzed, and distant metastasis was observed in $20(29.0 \%)$ patients [seven (19.4\%) in the consolidation group and $13(39.4 \%)$ in the control group]. The estimated 3-year DMFS was $81.91 \%$ in the consolidation group compared with $59.66 \%$ in the control group (HR: $0.4780 ; 95 \%$ CI: $0.2006-1.162 ; \mathrm{P}=0.10)$. The 3 -year OS was $88.15 \%$ in the consolidation group compared with $75.32 \%$ in the control group (HR: 0.5045; 95\% CI: 0.1658-1.594; $\mathrm{P}=0.25$ ). Although the estimated 3-year DMFS and OS were not significantly different between the two groups, the consolidation group exhibited a higher rate of DMFS and OS. Local recurrence was observed in $7(10.1 \%)$ patients overall [three $(8.3 \%)$ in the consolidation group and four (12.1\%) in the control group]. The estimated 3-year DFS was $85.48 \%$ in the consolidation group, and $56.64 \%$ in the control group (HR: 0.3992; 95\% CI: 0.1752-0.9366; $\mathrm{P}=0.036$, Fig. 3).

Surgical outcome and complications. All patients underwent optimal surgery with TME, among which 56 (73.6\%) patients in the consolidation group and $53(66.2 \%)$ patients 
Table I. Summary of patient characteristics.

\begin{tabular}{|c|c|c|c|c|}
\hline Parameter & $\begin{array}{c}\text { Total } \\
(n=156)\end{array}$ & $\begin{array}{l}\text { Consolidation } \\
\text { group }(n=76)\end{array}$ & $\begin{array}{c}\text { Control } \\
\text { group }(n=80)\end{array}$ & $\mathrm{P}$-value \\
\hline Age, median (range) & & $50(22-70)$ & $55(24-80)$ & 0.129 \\
\hline Sex & & & & - \\
\hline Male & $108(69.2 \%)$ & $57(75 \%)$ & $51(63.8 \%)$ & 0.128 \\
\hline Female & $48(30.8 \%)$ & $19(25 \%)$ & $29(36.3 \%)$ & - \\
\hline Clinical T-stage & & & & 0.130 \\
\hline cT3 & $37(23.7 \%)$ & $14(18.4 \%)$ & $23(28.8 \%)$ & - \\
\hline cT4 & $119(76.3)$ & $62(81.6 \%)$ & $57(71.2 \%)$ & - \\
\hline Clinical N-stage & & & & 0.539 \\
\hline cNO & $26(16.7 \%)$ & $14(18.4 \%)$ & $12(15.0 \%)$ & - \\
\hline $\mathrm{cN} 1$ & $49(31.4 \%)$ & $26(34.2 \%)$ & $23(28.7 \%)$ & - \\
\hline $\mathrm{cN} 2$ & $81(51.9 \%)$ & $36(47.4 \%)$ & $45(56.3 \%)$ & - \\
\hline AJCC/UICC stage & & & & 0.567 \\
\hline II & $26(16.7 \%)$ & $14(18.4 \%)$ & $12(15 \%)$ & - \\
\hline III & $130(83.3 \%)$ & $62(81.6 \%)$ & $68(85 \%)$ & - \\
\hline Differentiation & & & & 0.186 \\
\hline Well differentiated & $24(15.4 \%)$ & $10(13.2 \%)$ & $14(17.5 \%)$ & - \\
\hline Moderately differentiated & $89(57.1 \%)$ & $49(64.5 \%)$ & $40(50 \%)$ & - \\
\hline Poorly differentiated or mucinous adenocarcinoma & $43(27.5 \%)$ & $17(22.3 \%)$ & $26(32.5 \%)$ & - \\
\hline Distance from anorectal verge & & & & 0.307 \\
\hline $0-5 \mathrm{~cm}$ & $90(57.7 \%)$ & $47(61.8 \%)$ & $43(53.8 \%)$ & - \\
\hline $6-10 \mathrm{~cm}$ & $66(42.3 \%)$ & $29(31.2 \%)$ & $37(46.2 \%)$ & - \\
\hline Pretreatment with CEA & & & & 0.312 \\
\hline$<5 \mathrm{ng} / \mathrm{ml}$ & $88(56.4 \%)$ & $46(60.5 \%)$ & $42(52.3 \%)$ & - \\
\hline$\geq 5 \mathrm{ng} / \mathrm{ml}$ & $68(43.6 \%)$ & $30(39.5 \%)$ & $38(47.5 \%)$ & - \\
\hline Neural invasion & & & & 0.221 \\
\hline No & $146(93.5 \%)$ & $73(96.1 \%)$ & $73(91.3 \%)$ & - \\
\hline Yes & $10(6.5 \%)$ & $3(3.9 \%)$ & $7(8.7 \%)$ & - \\
\hline Lymph vascular invasion & & & & 0.959 \\
\hline No & $152(97.4 \%)$ & $74(97.4 \%)$ & $78(97.5 \%)$ & - \\
\hline Yes & $4(2.6 \%)$ & $2(2.6 \%)$ & $2(2.5 \%)$ & - \\
\hline Interval NCRT and TME & & & & 0.908 \\
\hline$\geq 8$ weeks & $89(57.1)$ & $43(56.6 \%)$ & $46(57.5 \%)$ & - \\
\hline$<8$ weeks & $67(42.9 \%)$ & $33(43.4 \%)$ & $34(42.5 \%)$ & - \\
\hline BMI & & & & 0.188 \\
\hline$\geq 25 \mathrm{~kg} / \mathrm{m}^{2}$ & $36(23.1 \%)$ & $21(27.6 \%)$ & $15(18.8 \%)$ & - \\
\hline$<25 \mathrm{~kg} / \mathrm{m}^{2}$ & $120(76.9 \%)$ & $55(72.4 \%)$ & $65(81.3 \%)$ & - \\
\hline Median follow-up time (months) & & 30 & 32 & 0.058 \\
\hline
\end{tabular}

AJCC, American Joint Committee on Cancer; UICC, Union for International Cancer Control 2010; CEA, carcinoembryonic antigen; NCRT, neoadjuvant chemoradiotherapy; TME, total mesorectal excision; BMI, body mass index.

in the control group received a sphincter-saving procedure $(\mathrm{P}=0.312)$. A total of $73(96.1 \%)$ patients in the consolidation group and $72(90.0 \%)$ patients in the control group obtained $\mathrm{R} 0$ resection of the primary tumor, with negative distal and radial margins. Comparing postoperative pathological findings with clinical status based on imaging prior to NCRT, a higher rate of downstaging for tumor $(65.8 \%$ vs. $48.6 \%, \mathrm{P}=0.032)$ and node $(55.3 \%$ vs. $35 \%, \mathrm{P}=0.011)$ category was observed in the consolidation group compared with the control group (Table III). No perioperative mortality occurred in this cohort. A total of 25/156 (16.02\%) patients developed postoperative complications, including intestinal obstruction $(6.5 \%$ vs. $7.5 \%$, $\mathrm{P}=0.822)$, anastomosis fistula $(1.31 \%$ vs. $1.25 \%, \mathrm{P}=0.971)$, infection $(6.57 \%$ vs. $2.5 \%, \mathrm{P}=0.219)$, urinary complications 
Table II. Multivariate analysis in relation to $\mathrm{pCR}+$ near $\mathrm{pCR}$ rate.

\begin{tabular}{|c|c|c|c|c|c|c|}
\hline Parameter & $\begin{array}{l}\text { No. of } \\
\text { patients }\end{array}$ & $\begin{array}{c}\mathrm{pCR}+\text { near } \\
\mathrm{pCR}^{\mathrm{a}}(\mathrm{N})\end{array}$ & $\begin{array}{c}\mathrm{pCR}+\text { near } \\
\mathrm{pCR}(\%)\end{array}$ & OR & $95 \% \mathrm{CI}$ & P-value \\
\hline Clinical T-stage & & & & 0.273 & $0.110-0.677$ & 0.005 \\
\hline cT3 & 37 & 16 & 43.2 & & & \\
\hline cT4 & 119 & 22 & 18.5 & & & \\
\hline Clinical N-stage & & & & 0.729 & $0.280-1.902$ & 0.518 \\
\hline $\mathrm{cN} 0$ & 26 & 12 & 46.1 & & & \\
\hline $\mathrm{cN} 1$ & 49 & 12 & 24.5 & & & \\
\hline $\mathrm{cN} 2$ & 81 & 14 & 17.3 & & & \\
\hline AJCC/UICC stage (\%) & & & & 0.260 & $0.041-1.661$ & 0.155 \\
\hline II & 26 & 12 & 46.1 & & & \\
\hline III & 130 & 26 & 20.0 & & & \\
\hline Pretreatment with CEA & & & & 0.378 & $0.151-0.944$ & 0.037 \\
\hline$<5 \mathrm{ng} / \mathrm{ml}$ & 88 & 28 & 31.8 & & & \\
\hline$\geq 5 \mathrm{ng} / \mathrm{ml}$ & 68 & 10 & 14.7 & & & \\
\hline Neural invasion & & & & 0.447 & $0.07-2.838$ & 0.393 \\
\hline No & 146 & 38 & 26.0 & & & \\
\hline Yes & 10 & 0 & 0.00 & & & \\
\hline Interval NCRT and TME & & & & 0.263 & $0.088-0.785$ & 0.017 \\
\hline$\geq 8$ weeks & 89 & 31 & 34.8 & & & \\
\hline$<8$ weeks & 67 & 7 & 10.4 & & & \\
\hline Given consolidation chemotherapy or not & & & & 0.378 & $0.151-0.944$ & 0.037 \\
\hline Consolidation group & 76 & 25 & 32.8 & & & \\
\hline Control group & 80 & 13 & 16.25 & & & \\
\hline
\end{tabular}

${ }^{a}$ Near pCR rate being defined by the finding of only isolated residual tumor cells or small groups of residual cancer cells in the rectal wall with no tumor cells in the lymph nodes. pCR, pathologic complete response; AJCC, American Joint Committee on Cancer; UICC, Union for International Cancer Control 2010; CEA, carcinoembryonic antigen; NCRT, neoadjuvant chemoradiotherapy; TME, total mesorectal excision; BMI, body mass index; Consolidation group, given neoadjuvant chemoradiotherapy and consolidation chemotherapy; Control group, given neoadjuvant chemoradiotherapy only.
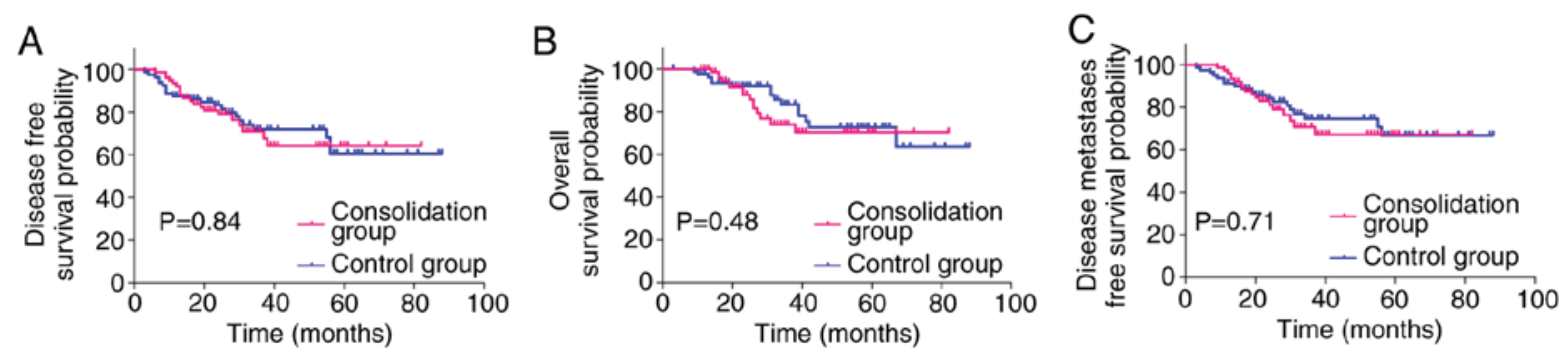

Figure 2. Kaplan-Meier curves presenting the 3-year DFS, OS and DMFS for patients with local advanced rectal cancer receiving consolidation chemotherapy (consolidation group), compared with patients not receiving consolidation chemotherapy (control group). (A) The 3-year DFS for the consolidation group was not significantly different compared with the control group $(\mathrm{P}=0.84)$. (B) The 3 -year OS was not significantly different for the consolidation group compared with the control group $(\mathrm{P}=0.48)$. (C) The 3-year DMFS was not significantly different for the consolidation group compared with the control group ( $\mathrm{P}=0.71)$. DFS, disease-free survival; OS, overall survival; DMFS, disease metastases free survival.

(0\% vs. $1.25 \%, \mathrm{P}=1.000)$, and pelvic fibrosis $(2.63 \%$ vs. $2.5 \%$, $\mathrm{P}=1.000)$. The incidence rate of complications did not differ significantly between the two groups (Table IV).

Toxicity. The grade 3 or 4 toxicities that developed during neoadjuvant treatment are described in Table $\mathrm{V}$. The rates of hematological toxicity and non-hematological toxicity were not influenced by the administration of consolidation chemotherapy. A total of nine (11.8\%) and six (7.5\%) patients in the consolidation and control groups had grade 3 or 4 leukopenia, respectively $(\mathrm{P}=0.358)$. Grade $3-4$ anemia was observed in four patients $(5.3 \%)$ in the consolidation group 
Table III. Summary of surgical outcome in the consolidation group compared with the control group.

\begin{tabular}{|c|c|c|c|}
\hline Parameter & Consolidation group, no. (\%) & Control group, no. (\%) & P-value \\
\hline No. of patients & 76 & 80 & - \\
\hline $\mathrm{pCR}+$ near $\mathrm{pCR}{ }^{\mathrm{a}}$ & $25(32.80)$ & $13(16.25)$ & 0.015 \\
\hline Anal preservation & $56(73.60)$ & $53(66.20)$ & 0.312 \\
\hline Resection with negative margins & $73(96.10)$ & $72(90.00)$ & 0.140 \\
\hline T downstaging (ypT<cT) & $50(65.8)$ & $39(48.6)$ & 0.032 \\
\hline $\mathrm{N}$ downstaging $(\mathrm{ypN}<\mathrm{cN})$ & $42(55.30)$ & $28(35.00)$ & 0.011 \\
\hline
\end{tabular}

${ }^{a}$ Near pCR rate being defined by the finding of only isolated residual tumor cells or small groups of residual cancer cells in the rectal wall with no tumor cells in the lymph nodes. pCR, pathologic complete response; T downstaging, tumor downstaging; $\mathrm{N}$ downstaging, node downstaging.
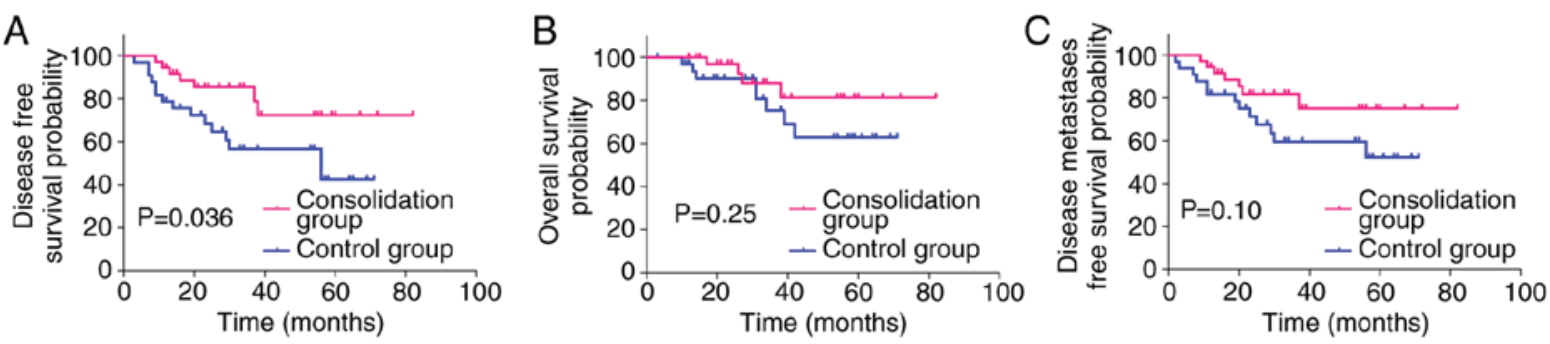

Figure 3. Kaplan-Meier curves presenting the 3-year DFS, OS and DMFS for patients with local advanced rectal cancer receiving consolidation chemotherapy (consolidation group), compared with patients not receiving consolidation chemotherapy (control group), in combination with standard adjuvant chemotherapy. (A) The 3-year DFS was significantly higher for the consolidation group compared with the control group (P=0.036). (B) The 3-year OS for the consolidation group was not significantly different compared with the control group $(\mathrm{P}=0.250)$. (C) The 3-year DMFS was not significantly different between the consolidation group and the control group $(\mathrm{P}=0.100)$. DFS, disease-free survival; OS, overall survival; DMFS, disease metastases free survival.

and in one patient $(1.3 \%)$ in the control group $(\mathrm{P}=0.155)$. Thrombocytopenia was observed in eight patients $(10.5 \%)$ and six patients $(7.5 \%)$ in the consolidation and control group, respectively $(\mathrm{P}=0.509)$. Overall, grade 3-4 nausea or vomiting, radiation proctitis, diarrhea, dermatitis, hand-foot syndrome, and peripheral neuropathy occurred in two $(2.6 \%)$, five $(6.6 \%)$, $15(19.7 \%)$, one $(1.3 \%)$, five $(6.6 \%)$, and two $(2.6 \%)$ patients in the consolidation group and in one $(1.3 \%, \mathrm{P}=0.530)$, four (5.0\%, $\mathrm{P}=0.67)$, nine $(11.3 \%, \mathrm{P}=0.142)$, one $(1.3 \%, \mathrm{P}=0.971)$, two $(2.5 \%, \mathrm{P}=0.219)$, and zero $(0 \%, \mathrm{P}=0.236)$ patients in the control group, respectively. Neither a life-threatening decrease in blood count nor febrile neutropenia was observed. No other grade 3 or 4 toxicities were recorded.

\section{Discussion}

Preoperative 45-50.4 Gy pelvic irradiation combined with fluorouracil or capecitabine monotherapy is the current conventional treatment regimen for LARC. However, a number of studies have reported that this treatment modality yields low pCR rate $(12-20 \%)$, and this treatment regimen has not significantly improved patients' survival $(5,6)$. Systemic metastasis is closely associated with long-term survival. However, the majority of patients with rectal cancer may have early micrometastases on initial diagnosis $(17,18)$. Previous studies have revealed that, although preoperative radiotherapy and TME surgery can improve the local control rate, they are inadequate for the treatment of systemic micrometastases $(17,18)$. Previous studies have also demonstrated that the later postoperative systemic chemotherapy was given, the higher the risk of postoperative metastases $(19,20)$. It has also been demonstrated that the addition of chemotherapy either before or following chemoradiotherapy may increase the pCR rate, indicating a synergistic response of additional chemotherapy to standard chemoradiation $(8,21)$. However, few studies regarding consolidation chemotherapy have been published, and even fewer studies concerning the survival of patients who underwent consolidation chemotherapy have been performed to date.

In the present study, a statistically significant increase in the rate of $\mathrm{pCR}$ and near $\mathrm{pCR}$ in patients administered consolidation chemotherapy compared with those administered conventional neoadjuvant treatment (32.8\% vs. $16.25 \%$, respectively), was observed, which largely coincides with the results of previous studies $(22,23)$. Habr-Gama et al (24) first reported the strategy of consolidation chemotherapy in the resting period between the completion of NCRT and surgery. In their cohort, $14(48 \%)$ patients achieved complete clinical response, and five (17\%) patients achieved pCR of the primary lesion following surgery. In 2015, Garcia-Aguilar et al (8) published a nonrandomized trial in which the addition of two to six cycles of infusional fluorouracil, leucovorin, and oxaliplatin was associated with an increased pCR rate (18-38\%), without increasing complications, compared with the strategy of traditional NCRT in patients with LARC. However, there was a major limitation to this study; as the number of cycles of consolidation chemotherapy increased, the interval between neoadjuvant therapy and surgery also 
Table IV. Summary of complications in the consolidation group compared with the control group.

\begin{tabular}{lccc}
\hline Parameter & Consolidation group, no. $(\%)$ & Control group, no. $(\%)$ & P-value \\
\hline No. of patients & 76 & 80 & - \\
Intestinal obstruction & $5(6.50)$ & $6(7.50)$ & 0.822 \\
Anastomotic fistula & $1(1.31)$ & $1(1.25)$ & 0.971 \\
Infection & $5(6.57)$ & $2(2.50)$ & 0.219 \\
Urinary complications & $0(0.00)$ & $1(1.25)$ & 1.000 \\
Pelvic fibrosis & $2(2.63)$ & $2(2.50)$ & 1.000 \\
\hline
\end{tabular}

Table V. Preoperative chemoradiotherapy grade 3 or 4 toxicities in the consolidation group compared with the control group.

\begin{tabular}{|c|c|c|c|}
\hline Parameter & Consolidation group, no. (\%) & Control group, no. (\%) & P-value \\
\hline No. of patients & 76 & 80 & - \\
\hline \multicolumn{4}{|l|}{ Hematologic toxicity } \\
\hline Leukopenia & $9(11.8)$ & $6(7.5)$ & 0.358 \\
\hline Anemia & $4(5.3)$ & $1(1.3)$ & 0.155 \\
\hline Thrombocytopenia & $8(10.5)$ & $6(7.5)$ & 0.509 \\
\hline \multicolumn{4}{|l|}{ Nonhematologic toxicity } \\
\hline Nausea or vomiting & $2(2.6)$ & $1(1.3)$ & 0.530 \\
\hline Radiation proctitis & $5(6.6)$ & $4(5.0)$ & 0.67 \\
\hline Diarrhea & $15(19.7)$ & $9(11.3)$ & 0.142 \\
\hline Dermatitis & $1(1.3)$ & $1(1.3)$ & 0.971 \\
\hline Hand-foot syndrome & $5(6.6)$ & $2(2.5)$ & 0.219 \\
\hline Peripheral neuropathy & $2(2.6)$ & $0(0.0)$ & 0.236 \\
\hline
\end{tabular}

increased, which may lead to a high pCR rate. Nonetheless, it has been demonstrated that the increased $\mathrm{pCR}$ rate may depend on the timing of response assessment (15). In the present study, the median time interval between the two groups was 8.7 weeks in the consolidation group and 7.4 weeks in the control group $(\mathrm{P}=0.218)$. The majority of patients $(72 \%)$ received two cycles of consolidation chemotherapy, which may reduce the impact of the number of cycles and the treatment interval on pCR and near pCR. Consolidation chemotherapy may improve the $\mathrm{pCR}$ rate, and this may be due to numerous reasons. Firstly, extending chemotherapy to the resting period may also allow further exposure of irradiated tumor cells to chemotherapy and eradicate the minimal residual tumor cells (24). Additionally, administering preoperative chemotherapy is conducive to further reducing the tumor burden, as the tumor has a rich blood supply in the preoperative phase, which is beneficial to the distribution of chemotherapy drugs. Lastly, the general condition of the patient is likely to be improved, and thus chemotherapy is better tolerated at this stage.

Data on the impact of consolidation chemotherapy on long-term disease recurrence and survival are limited. The present study has demonstrated an improved DFS $(85.48 \%$ vs. $56.64 \%, \mathrm{P}=0.036)$ in patients administered consolidation chemotherapy following stratified analysis by selecting patients with $>3$ months of adjuvant chemotherapy. OS and
DMFS were not significantly different between the two groups, although the consolidation group displayed a higher rate of DMFS and OS (81.91\% vs. $59.66 \%, \mathrm{P}=0.10 ; 88.15 \%$ vs. $75.32 \%, \mathrm{P}=0.25)$ compared with conventional NCRT. The results from the present study verified that consolidation chemotherapy may be associated with improved local control rate and favorable DFS. It may represent a subgroup of LARC with an improved biological behavior. Larsen et al (25) evaluated the prognostic value of CapeOX regimen before, during and after NCRT in LARC and reported a 5-year OS and PFS of 72 and 62\%, respectively. Garcia-Aguilar et al (8) recently published a nonrandomized trial in which the addition of 2-6 cycles of FOLFOX was associated with an increased pCR rate (from 18-38\%), however the study did not provide evidence of a benefit to long-term survival.

One concern with the use of consolidation chemotherapy is that it may significantly increase toxicity, reduce tolerance and compromise the treatment effect. In the present study, consolidation chemotherapy was well tolerated. Grade 3-4 toxicities were also comparable with those using conventional NCRT (8). This result may be attributed to the following factors: Firstly, modern, highly conformal radiation therapy planning and delivery techniques may potentially reduce the radiation dose to the bowel and pelvis, consequently reducing gastrointestinal side effects and hematological toxicity (26); Secondly, consolidation chemotherapy may be better tolerated 
following NCRT, as patients have not been affected by surgical trauma or complications (27).

In the present study, the $\mathrm{R} 0$ resection rate of patients in the consolidation chemotherapy group was $96.1 \%$. This corresponds to study of Gao et al (23). Engineer et al (28) previously demonstrated that the addition of chemotherapy following neoadjuvant radiochemotherapy for patients with poor or no response to NCRT may achieve a considerable R0 resection rate $(62 \%)$. A modest increase in the rate of $\mathrm{R} 0$ resection in patients treated with consolidation chemotherapy compared with those treated with traditional neoadjuvant chemoradiotherapy ( $96.1 \%$ vs. $90 \%$, respectively), was observed in the present study; however, it did not reach statistical significance $(\mathrm{P}=0.14)$. This may be a result of different attending surgeons and the small sample size of the study. The present study demonstrated an increase in the rate of anal preservation in patients administered consolidation chemotherapy, although the increase also did not reach statistical significance (73.6\% vs. $66.2 \%$; $\mathrm{P}=0.321$ ).

The present study poses a number of limitations. Firstly, as it is a retrospective study, it may have certain disadvantages, including potential selection bias, recall bias and censored data. However, considering the lack of prospectively collected data available, the results may be interpreted as clinically significant. Secondly, owing to the paucity of conclusive data on the use of chemotherapy regimens in patients with stage II/III rectal cancer, FOLFOX, CapeOx, 5-FU/leucovorin or capecitabine are all recommended by the NCCN panel, therefore the protocols of consolidation chemotherapy included in the present study are somewhat heterogeneous. Thirdly, although the results appeared promising, the cohort was too small and the follow-up period too short to confirm whether this strategy yields an improved survival benefit compared with conventional NCRT. Due to the limited sample size and the short follow-up period, it was not possible to adequately analyze all of the results. Therefore, further investigations are required.

In conclusion, this preliminary study revealed that consolidation chemotherapy administered between NCRT and surgery may be associated with improved rates of $\mathrm{pCR}$ and near $\mathrm{pCR}$, TN downstaging and DFS. Furthermore, the addition of chemotherapy did not lead to higher toxicity, complications, or worse quality of mesorectal excision. Consolidation chemotherapy is safe and associated with improved oncological outcomes, and may reduce the number of required cycles of postoperative chemotherapy or eliminate the need for such a treatment modality.

\section{Acknowledgements}

Not applicable.

\section{Funding}

This study was supported by National Natural Science Foundation of China (grant nos. 81672756 and 81472711).

\section{Availability of data and material}

The datasets used and/or analyzed during the current study are available from the corresponding author on reasonable request.

\section{Authors' contributions}

HQL and DHW conceived and designed the experiments. HQL and PYL collected the data. JL performed the pathological evaluation. HQL and QZ conducted the clinical follow-up. ZJL and ZYD performed the statistical analyses. HQL and ZYD drafted and revised the manuscript. All authors read and approved the final manuscript.

\section{Ethics approval and consent to participate}

The Ethics Committee of Nanfang Hospital of Southern Medical University (Guangzhou, China) approved the present study.

\section{Patient consent for publication}

Not applicable.

\section{Competing interests}

The authors declare that they have no competing interests.

\section{References}

1. Bray F, Sankila R, Ferlay J and Parkin DM: Estimates of cancer incidence and mortality in Europe in 1995. Eur J Cancer 38: 99-166, 2002.

2. Heald RJ, Husband EM and Ryall RD: The mesorectum in rectal cancer surgery-the clue to pelvic recurrence? Br J Surg 69: 613-616, 1982 .

3. Havenga K, Enker WE, Norstein J, Moriya Y, Heald RJ, van Houwelingen $\mathrm{HC}$ and van de Velde CJ: Improved survival and local control after total mesorectal excision or D3 lymphadenectomy in the treatment of primary rectal cancer: An international analysis of 1,411 patients. Eur J Surg Oncol 25: 368-374, 1999.

4. Benson AB, Venook AP, Cederquist L, Chan E, Chen YJ, Cooper HS, Deming D, Engstrom PF, Enzinger PC, Fichera A and Grem JL: Colon cancer, version 1.2017, NCCN clinical practice guidelines in oncology. J Natl Comp Cancer Network. 15: 370-398, 2017.

5. Chua YJ: Pathological complete response: Still a relevant endpoint in rectal cancer? The Lancet Oncology 11: 807-808, 2010.

6. Maas M, Nelemans PJ, Valentini V, Das P, Rödel C, Kuo LJ, Calvo FA, García-Aguilar J, Glynne-Jones R, Haustermans $\mathrm{K}$, et al: Long-term outcome in patients with a pathological complete response after chemoradiation for rectal cancer: A pooled analysis of individual patient data. Lancet Oncol 11: 835-844, 2010.

7. Francois Y, Nemoz CJ, BaulieuxJ, Vignal J, Grandjean JP, Partensky C, Souquet JC, Adeleine P and Gerard JP: Influence of the interval between preoperative radiation therapy and surgery on downstaging and on the rate of sphincter-sparing surgery for rectal cancer: The Lyon R90-01 Randomized Trial. J Clin Oncol 17: 2396, 1999.

8. Garcia-Aguilar J, Chow OS, Smith DD, Marcet JE, Cataldo PA, Varma MG, Kumar AS, Oommen S, Coutsoftides T, Hunt SR, et al: Effect of adding mFOLFOX6 after neoadjuvant chemoradiation in locally advanced rectal cancer: A multicentre, phase 2 trial. Lancet Oncol 16: 957-966, 2015.

9. Tulchinsky H, Shmueli E, Figer A, Klausner JM and Rabau M: An interval $>7$ weeks between neoadjuvant therapy and surgery improves pathologic complete response and disease-free survival in patients with locally advanced rectal cancer. Ann Surg Oncol 15: 2661-2667, 2008.

10. Swellengrebel HA, Bosch SL, Cats A, Vincent AD, Dewit LG, Verwaal VJ, Nagtegaal ID and Marijnen CA: Tumour regression grading after chemoradiotherapy for locally advanced rectal cancer: A near pathologic complete response does not translate into good clinical outcome. Radiother Oncol 112: 44-51, 2014. 
11. Wolthuis AM, Penninckx F, Haustermans K, De Hertogh G Fieuws S, Van Cutsem E and D'Hoore A: Impact of interval between neoadjuvant chemoradiotherapy and TME for locally advanced rectal cancer on pathologic response and oncologic outcome. Ann Surg Oncol 19: 2833-2841, 2012.

12. Habr-Gama A, Perez RO, Proscurshim I, Nunes Dos Santos RM, Kiss D, Gama-Rodrigues J and Cecconello I: Interval between surgery and neoadjuvant chemoradiation therapy for distal rectal cancer: Does delayed surgery have an impact on outcome? Int J Radiat Oncol Biol Phys 71: 1181-1188, 2008.

13. Supiot S, Bennouna J, Rio E, Meurette G, Bardet E, Buecher B, Dravet F, Le Neel JC, Douillard JY, Mahé MA and Lehur PA: Negative influence of delayed surgery on survival after preoperative radiotherapy in rectal cancer. Colorectal Dis 8: 430-435 2006.

14. Edge SB and Compton CC: The american joint committee on cancer: The 7th edition of the AJCC cancer staging manual and the future of TNM. Ann Surg Oncol 17: 1471-1474, 2010.

15. Moore HG, Gittleman AE, Minsky BD, Wong D, Paty PB, Weiser M, Temple L, Saltz L, Shia J, Shia J and Guillem JG: Rate of pathologic complete response with increased interval between preoperative combined modality therapy and rectal cancer resection. Dis Colon Rectum 47: 279-286, 2004.

16. Patel UB, Taylor F, Blomqvist L, George C, Evans H, Tekkis P, Quirke P, Sebag-Montefiore D, Moran B, Heald R, et al: Magnetic resonance imaging-detected tumor response for locally advanced rectal cancer predicts survival outcomes: MERCURY experience. J Clin Oncol 29: 3753-3760, 2011.

17. Sauer R, Becker H, Hohenberger W, Rodel C, Wittekind C, Fietkau R, Martus P, Tschmelitsch J, Hager E, Hess CF, et al: Preoperative versus postoperative chemoradiotherapy for rectal cancer. N Engl J Med 351: 1731-1740, 2004.

18. Cienfuegos JA, Baixauli J, Pastor C, Arredondo J, Sola JJ, Arbea L, Chopitea A and Hernandez-Lizoain JL: Long-term oncologic results in cancer of the rectum treated by preoperative chemoradiotherapy and surgery: An analysis of 500 cases. Rev Esp Enferm Dig 107: 340-346, 2015.

19. Lima IS, Yasui Y, Scarfe A and Winget M: Association between receipt and timing of adjuvant chemotherapy and survival for patients with stage III colon cancer in Alberta, Canada. Cancer 117: 3833-3840, 2011

20. Tevis SE, Kohlnhofer BM, Stringfield S, Foley EF, Harms BA, Heise CP and Kennedy GD: Postoperative complications in patients with rectal cancer are associated with delays in chemotherapy that lead to worse disease-free and overall survival. Dis Colon Rectum 56: 1339-1348, 2013.
21. Chau I, Brown G, Cunningham D, Tait D, Wotherspoon A, Norman AR, Tebbutt N, Hill M, Ross PJ, Massey A and Oates J: Neoadjuvant capecitabine and oxaliplatin followed by synchronous chemoradiation and total mesorectal excision in magnetic resonance imaging-defined poor-risk rectal cancer. J Clin Oncol 24: 668-674, 2006.

22. Gao YH, Zhang X, An X, Cai MY, Zeng ZF, Chen G, Kong LH, Lin JZ, Wan DS, Pan ZZ and Ding PR: Oxaliplatin and capecitabine concomitant with neoadjuvant radiotherapy and extended to the resting period in high risk locally advanced rectal cancer. Strahlenther Onkol 190: 158-164, 2014.

23. Gao YH, Lin JZ, An X, Luo JL, Cai MY, Cai PQ, Kong LH, Liu GC, Tang JH, Chen G, et al: Neoadjuvant sandwich treatment with oxaliplatin and capecitabine administered prior to, concurrently with, and following radiation therapy in locally advanced rectal cancer: A prospective phase 2 trial. Int J Radiat Oncol Biol Phys 90: 1153-1160, 2014.

24. Habr-Gama A,Perez RO, Sabbaga J, Nadalin W, São Julião GP and Gama-Rodrigues J: Increasing the rates of complete response to neoadjuvant chemoradiotherapy for distal rectal cancer: Results of a prospective study using additional chemotherapy during the resting period. Dis Colon Rectum 52: 1927-1934, 2009.

25. Larsen FO, Markussen A, Jensen BV, Fromm AL, Vistisen KK, Parner VK, Linnemann D, Hansen RH, Johannesen HH and Schou JV: Capecitabine and oxaliplatin before, during, and after radiotherapy for High-Risk rectal cancer. Clin Colorectal Cancer 16: e7-e14, 2017.

26. Wan J, Liu K, Li K, Li G and Zhang Z: Can dosimetric parameters predict acute hematologic toxicity in rectal cancer patients treated with intensity-modulated pelvic radiotherapy? Radiat Oncol 10: 162, 2015

27. Helbling D, Bodoky G, Gautschi O, Sun H, Bosman F, Gloor B, Burkhard R, Winterhalder R, Madlung A, Rauch D, et al: Neoadjuvant chemoradiotherapy with or without panitumumab in patients with wild-type KRAS, locally advanced rectal cancer (LARC): A randomized, multicenter, phase II trial SAKK 41/07. Ann Oncol 24: 718-725, 2013.

28. Engineer R, Ostwal V, Arya S, Gupta P, Chopra S, Patil P, Jatal S and Saklani A: Additional chemotherapy and salvage surgery for poor response to chemoradiotherapy in rectal cancers. Asia Pac J Clin Oncol 13: 322-328, 2017.

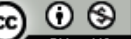

This work is licensed under a Creative Commons Attribution 4.0 International (CC BY-NC 4.0) License 